\title{
Descending necrotising mediastinitis
}

\author{
Hassam Alsoub, Kadavil C Chacko
}

\begin{abstract}
Summary
Descending necrotising mediastinitis is a rare but serious complication of oropharyngeal infections with high mortality. Diagnosis is frequently delayed, contributing to this high mortality, but awareness of such a complication and early diagnosis using computed tomographic scanning leads to prompt surgical drainage, proper antibiotic therapy, and survival.
\end{abstract}

Keywords: descending necrotising mediastinitis, CT scanning

\section{Introduction}

Acute suppurative mediastinitis is a severe infectious condition that is most commonly the result of oesophageal perforation or rupture. Oropharyngeal infections may cause descending necrotising mediastinitis characterised by rapid tissue destruction and high mortality unless vigorous and effective therapeutic measures are taken. Three cases of descending necrotising mediastinitis secondary to peritonsillar abscess have been reported previously. Over the last two years we have treated two patients with descending necrotising mediastinitis secondary to peritonsillar abscess. The clinical courses of these two patients are described and previous publications reviewed.

Case 1

A 32-year-old previously healthy man presented with a four-day history of sore throat, difficulty in swallowing, and fever. Physical examination revealed a pyrexia of $40.8^{\circ} \mathrm{C}$, blood pressure of $90 / 60 \mathrm{mmHg}$ and a pulse rate of 135 beats/min. Examination of the throat revealed a right peritonsillar abscess, but otherwise physical examination was normal. Laboratory investigation on admission revealed a haemoglobin of $13.0 \mathrm{~g} / \mathrm{dl}$, white blood cell count of $7.6 \times 10^{9} / 1$, and platelets $14.7 \times 10^{9} / 1$. Liver function tests, serum creatinine and chest $\mathrm{X}$-ray were normal. Incision of the abscess yielded a small amount of bloody fluid but no culture was done. The patient was started on intravenous cephalothin $500 \mathrm{mg}$ every six hours. On the fifth hospital day he developed rapidly increasing swelling involving both sides of the neck and supraclavicular regions with crepitus. A computed tomographic (CT) scan of the chest demonstrated an abscess with gas collection in the anterior mediastinum down to the level of the heart, bilateral pleural effusion, and pericardial effusion (figures 1 and 2). Cephalothin was discontinued and intravenous imipenem/ cilastatin $500 \mathrm{mg}$ every six hours was started, and surgical drainage was carried out through a transcervical incision. Culture of the pus grew Streptococcus viridans. Two days later chest $\mathrm{X}$-ray showed further widening of the mediastinum, a large left pleural effusion, and an echocardiogram showed a large pericardial effusion. Mediastinal drainage was done again through a subxiphoid incision and pericardial and left pleural tubes were inserted yielding purulent fluids. The patient's condition then improved gradually and the mediastinal drain, pericardial, and left pleural tubes were removed on the 20th hospital day. Imipenem/ cilastatin was continued for total of six weeks; the patient was then discharged home in good health.

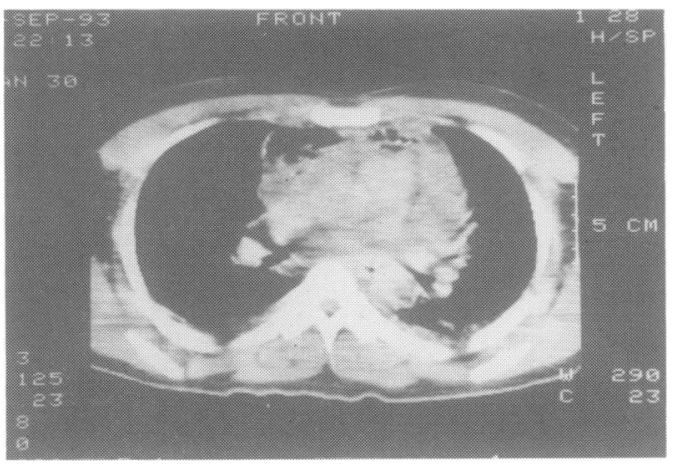

Figure 1 CT scan at the level of larynx showing extensive gas-forming infection involving both sides of the neck

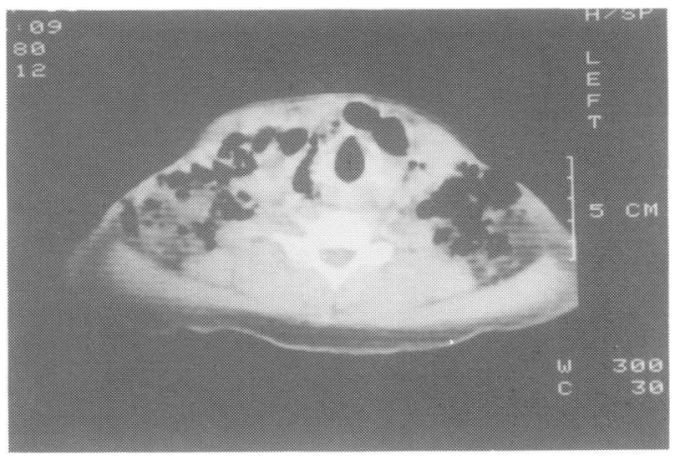

Figure 2 CT scan at the level of the heart showing extension of the infection to the anterior mediastinum and pericardium 
Case 2

A 47-year-old man with insulin-dependent diabetes mellitus was admitted with a 24-h history of throat pain and trismus. Physical examination revealed a temperature of $37.9^{\circ} \mathrm{C}$, pulse rate of 104 beats/min and blood pressure $210 / 120 \mathrm{mmHg}$. Throat examination revealed a right peritonsillar abscess. Laboratory investigation on admission revealed: a haemoglobin $12.3 \mathrm{~g} / \mathrm{dl}$, white blood cell count $16.4 \times 10^{9} / \mathrm{l}$, platelet $30.4 \times 10^{9} / 1$, random blood sugar $14.6 \mathrm{mmol}$, serum creatinine $220 \mu \mathrm{mol} / \mathrm{l}$, and normal chest X-ray film. He was started on intravenous cephalothin $1.5 \mathrm{~g}$ every six hours with slight improvement. On the sixth hospital day incision of the abscess was carried out but only a small amount of blood was obtained. Intravenous clindamycin (600 mg every six hours) was added. On the 11 th hospital day he developed swelling in the anterior aspect of the neck and both supraclavicular regions, more in the right side, with no crepitus. A CT scan of neck and chest demonstrated a right peritonsillar abscess $3 \mathrm{~cm}$ in diameter and an abscess extending from the left oropharynx down to the mediastinum anterior to the trachea and behind the arch of the aorta with air bubbles (figures 3, 4). Transcervical draining of the mediastinum was done, but culture of the pus failed to grow any organism. After that the patient continued to improve gradually. Imipenem/cilastatin was continued for a total of 30 days, then he was discharged home in good health.

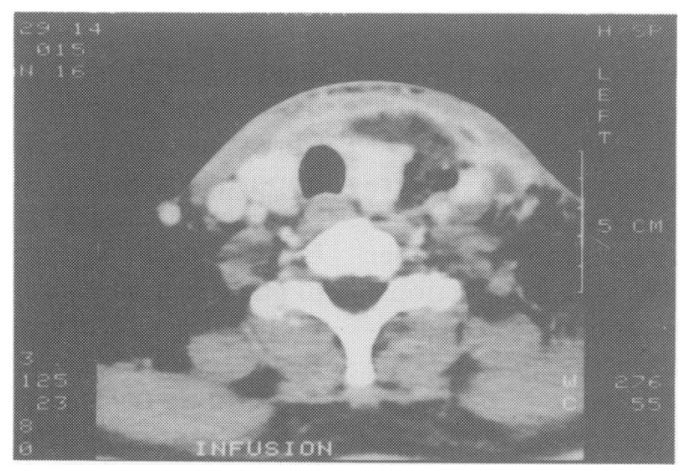

Figure 3 Post-contrast CT scan of the neck showing an abscess in the left para-pharyngeal space with ring enhancement

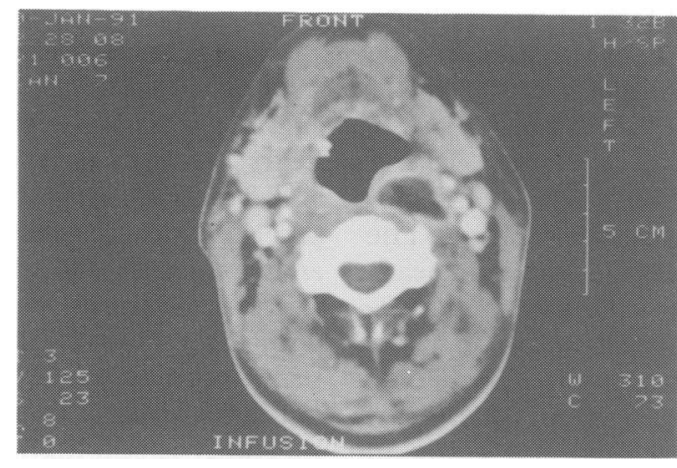

Figure 4 Post-contrast CT scan at the level of the thyroid gland, showing extension of the infection downwards with the gas formation

\section{Discussion}

Acute mediastinitis is an uncommon condition; it is mostly seen as a complication of oesophageal perforation or following cardiac surgery. Acute mediastinitis unrelated to surgical procedures was considered a rare infection even in the pre-antibiotic era. ${ }^{1}$ Today, liberal and early antibiotic utilisation has made this condition even more rare, but it is still seen as a complication of infection in the oropharynx, or second and third mandibular molar with dissection downward along the anatomic fascial planes to the mediastinum. Such a variety is called descending necrotising mediastinitis. Pearse ${ }^{2}$ in 1938 reported one of the first series of patients with mediastinitis following orodental infections. He described 100 patients, 64 of whom were the result of oesophageal perforation while only 21 were the result of oropharyngeal infection and in this group he reported a mortality of more than $50 \%$. Over 50 further cases of descending necrotising mediastinitis have now been reported..$^{3-28} \mathrm{We}$ adopted the criteria proposed by Estrera $e \mathrm{al}^{3}$ for the diagnosis of descending necrotising mediastinitis (see box).

The group consisted of 36 males, 10 females and five patients for whom sex was not specified. Ages ranged from one month to 64 years with an average of 33.5 years. The most common cause was odontogenic infection usually arising from second and third mandibular molars (table). Patients with acute mediastinitis are often severely ill (see box overleaf). Most recent studies have emphasized the polymicrobial nature of these infections., ${ }^{2,3}$ The organisms involved in descending necrotising mediastinitis are usually mixed aerobes and anaerobes, accounting for $47 \%$ of cases, aerobes only (usually B-haemolytic streptococcus) account for $23 \%$ and anaerobes only $30 \%$ of cases. ${ }^{3}$ These usually reflect the organisms present in the mouth. The synergistic action of

\section{Criteria for diagnosis of descen-} ding necrotising mediastinitis

- clinical manifestation of severe infection

- characteristic X-ray features of mediastinitis

- necrotising mediastinal infection at operation or post-mortem

- relationship to oropharyngeal infection, with the development of the necrotising process

Table Conditions causing descending necrotising mediastinitis

\begin{tabular}{lc}
\hline Cause & No of patients \\
\hline Odontogenic infection & 31 \\
Retropharyngeal abscess & 6 \\
Peritonsillar abscess & 3 \\
Cervical lymphadenitis & 2 \\
Trauma & 3 \\
Endotracheal intubation & 5 \\
Unknown & 1 \\
Total & 51 \\
\hline
\end{tabular}


Descending necrotising mediastinitis

Clinical features

- severely ill

- fever, tachycardia

- oedema of neck or chest wall with crepitus

- purulent pleural and/or pericardial effusion

aerobic and anaerobic organisms may explain the virulence of these infections.

In the evaluation of cases of descending necrotising mediastinitis the use of X-ray examination of the neck and chest is not very helpful. It may show widening of the mediastinum with gas bubbles, however, these findings are usually late in the course of the disease. CT scan has proved to be a very useful aid in the diagnosis of descending necrotising mediastinitis and its management. It allows early diagnosis of the disease with great accuracy and confidence; it aids in the choice of the surgical approach to drain the mediastinum and in the follow-up of patients after drainage.

The management of patients with descending necrotising mediastinitis consist of an adequate surgical drainage combined with proper antibiotic choice. Because these infections are usually poly-microbial with both aerobic and anaerobic organisms, the initial antibiotic choice should cover these organisms and antibiotic combinations are often required. Later on when the culture results are available antibiotics can be changed accordingly. To await the results of the culture and sensitivity studies prior to initiation of antimicrobial therapy, a common practice for other infections, is dangerous and ill-advised.

A thorough knowledge of the interrelationships between the fascial spaces of the neck and mediastinum is crucial to the proper surgical management of these infections. Several authors have written detailed descriptions of the anatomy of this region and the reader is referred to one of them. ${ }^{2,36} \mathrm{~A}$ few key points will be made. There are three primary routes of spread of infection from the neck to the mediastinum. The pretracheal space lies anterior to the trachea and posterior to the strap muscles and pretracheal fascia; its superior limit is the thyroid cartilage and it descends into the anterior mediastinum. At the level of the carina the pretracheal fascia fuses with the pericardium and parietal pleura, explaining the frequent association of empyema and pericardial effusion with anterior mediastinal abscess; spread of infection along this route is not common, accounting for only $7 \%$ of cases of descending necrotising mediastinitis. The perivascular space includes the carotid sheath and its neural and vascular structures. Involvement of this space may result in major vessel rupture and cranial nerve deficits. Spread of infection along this space accounts for about $20 \%$ of cases. The most frequent route of spread of descending infections into the mediastinum is through the retrovisceral space, accounting for about $71 \%$ of cases. ${ }^{13}$ This space extends from the skull base inferiorly into the posterior mediastinum to the diaphragm. Infections of the second and third mandibular molars may result in submandibular triangle abscess. By extension of the abscess beyond the posterior limit of the mylohyoid shelf, involvement of the retrovisceral space can occur with subsequent involvement of the mediastinum. Spread of infection from the oropharynx to the mediastinum is enhanced by gravity and the negative intrathoracic pressure.

The standard and most commonly used approach to drain the mediastinum is the trans-cervical approach. However, many authorities in this field believe that this approach may be inadequate and even may delay definitive operation to drain the mediastinum. ${ }^{3,4}$ They recommend an approach which depends on the CT scan finding at the time of diagnosis. If the superior mediastinum only is involved and the infection is contained above the level of the fourth thoracic vertebra posteriorly or tracheal bifurcation anteriorly, mediastinal drainage may be accomplished by the trans-cervical approach. However, in patients with infection below this level, mediastinal drainage is best accomplished by subxiphoid or trans-thoracic drainage, in addition to drainage of their cervical infections. Drainage of the pericardial and pleural spaces is also necessary if they are involved. The results of the treatment of our patients further support these recommendations. Our first patient had extensive disease with extension below the fourth thoracic vertebra with pleural and pericardial effusion. In this patient transcervical drainage was not adequate and he needed subxiphoid drainage, while the second patient had limited disease and in this patient trans-cervical drainage was adequate. An important point in the management of these patients is the use of tracheostomy, which could be an integral part of their treatment. The use of an endotracheal tube is discouraged because of the risks of reintubation. ${ }^{29}$ Several complications have been reported in patients with descending necrotising mediastinitis which include compromise of pulmonary function by fluid accumulating in the pleural and extrapleural spaces, exsanguination from vessel erosion, cranial nerve palsies (IX, X, XII), and epidural abscess. ${ }^{30-32}$

The mortality rate from descending necrotising mediastinitis remains high, the reasons for this are: firstly, these infections tend to be rapidly spreading and accompanied by a fulminant sepsis. Secondly, there is often significant delay before the diagnosis is made. Pearse $^{2}$ has reported a mortality of more than $50 \%$ among 21 patients with mediastinitis secondary to oropharyngeal infections. Estrera et $a l^{3}$ in 1983 reported a mortality of $42 \%$, however, since his report, another 20 patients with descending necrotising mediastinitis have been reported, 5,25-28,33-35 including our two patients, with $25 \%$ mortality. Although this mortality is still high, however, it represents a $17 \%$ drop from that reported by Estrera et al. The reasons for this drop are multifactorial, an important one being the use of CT scanning 
which allows early diagnosis of descending necrotising mediastinitis and initiation of treatment. The usefulness of CT scanning was first noticed by Estrera et al who attributed the survival of three of his last four patients to early diagnosis using CT. Other reasons that could have contributed to this drop in mortality include a surgical approach that more often uses the subxiphoid incision or thoracotomy and not only the transcervical incision to drain the mediastinum, the use of modern antibiotics, and intensive care management.

\section{Conclusion}

Descending nectrotising mediastinitis secondary to oropharyngeal infection is rare. The mortality is $25 \%$ for cases reported in the last 10 years. The treatment depends on accurate and prompt diagnosis. CT scanning is the single most important tool for the early diagnosis of descending necrotising mediastinitis. Early and complete mediastinal drainage via

1 Myers J. The chest and heart. In: Myers J, McKinlay C, eds. Springfield, Illinois: Charles C Thomas, 1948; vol 1: pp 265-6.

2 Pearse HE Jr. Mediastinitis following cervical suppuration. Ann Surg 1938; 107: 588-611.

3 Estrera AS, Landy MJ, Glusham JM, Sinn DP, Platt MR. Descending necrotizing mediastinitis. Surg Gynecol Obstet 1983; 157: 545-52.

4 Wheatly MJ, Stirling MC, Kirsh MM, Gago O, Oringer MB. Descending necrotizing mediastinitis: transcervical

drainage is not enough. Ann Thorac Surg 1990; 49: 780-4.

Zachariades N, Mczttis M, Starrinidis P, KonsolokAgouridak E. Mediastinitis thoracic empyema and pericarditis as complication of

6 McCurdy JA Jr, MacInnis EL, Hayes LL. Fatal mediastinitis after a dental infection. F Oral Surg 1977; 35: 726-9. 7 Janecka IP, Rankow RM. Fatal mediastinitis following retropharyngeal abscess. Arch Otolaryngol 1971: 93: 630-3. 8 Howell HS, Prinz RA, Pickleman JR. Anaerobic mediastinitis. Surg Gynecol Obstet 1976; 43: 353-9.

9 Hendler BH, Quinn PD. Fatal mediastinitis secondary to odontogenic infection. $\mathcal{f}$ Oral Surg 1978; 36: 308-10.

10 Economopoulos GC, Scherzer HH, Gryboski WA. Successful management of mediastinitis, pleural empyema, and aorto-pulmonary fistula from odontogenic infection. Ann Thorac Surg 1983; 35: 184-7.

11 Strauss HR, Tilghman DM, Hankins J. Ludwig angina, empyema, pulmonary infiltration, and pericarditis secondary to extraction of a tooth. F Oral Surg 1980; 38: 223-9.

12 Wills PI, Vernon RP. Complications of space infections of head and neck. Laryngoscope 1981; 91: 1129-36.

13 Moncada R, Warpeha R, Pickleman J, et al. Mediastinitis from odontogenic and deep cervical infection. Chest 1978; 73: 497-500.

4 Enquist RW, Blanck RR, Butler RH. Nontraumatic mediastinitis. $\mathcal{F} A M A$ 1976; 236: $1048-9$.

15 Scully RE, Galdabini JJ, McNeely BU, Meade RH. Case records of the Massachusetts General Hospital (case 151978). N Engl f Med 1978; 298: 894-902.

16 North J, Emanuel B. Mediastinitis in a child caused by perforation of the pharynx. Am $₹$ Dis Child 1975; 129: $962-4$

17 Brooks V. Suppurative soft tissue infection of the head and neck. West Indian Med F 1963; 12: 200-12.

18 Hawkins DB, Seltzer DC, Barnett TE, Stoneman GB. Endotracheal tube perforation of the hypopharynx. West $\dot{\mathcal{f}}$ Med 1974; 120: 282-6.
Descending necrotising mediastinitis

- rare

- oedema of neck and chest wall, with crepitus, are the classical signs

- CT scanning is useful in early diagnosis

- mediastinal drainage and antibiotic therapy are the treatment of choice

- mortality $25 \%$

trans-cervical incision or using a thoracotomy or subxiphoid incision, along with antibiotic therapy, provide the basis for treatment in these patients.

The authors are indebted to Mr Kochuplavilayil Daniel John for his secretarial help in the preparation of the manuscript.

19 Wolffa AP, Kuhn FA, Ogura JH. Pharyngeal-esophageal perforations associated with rapid oral endotracheal intubation. Ann Otol Rhinol Laryngol 1972; 81: 258-61.

20 Richardson JD, Fox GL, Grover FL, Crux AB Jr. Necrotizing fasciitis of the neck. Tex Med f 1975; 71: 69-71.

21 Albertsen J, Thomsen EM. Nonclostridial deep gasproducing infection in the neck. Arch Otolaryngol 1970; 92: 383-5.

22 Cogan MIC. Necrotizing mediastinitis secondary to descending cervical cellulitis. Oral Surg 1973; 36: 307-20.

23 Young JN, Samson PC. Extrapleural empyema thoracis as a direct extension of Ludwig's angina. $\mathcal{F}$ Thorac Cardiovasc Surg 1980; 80: 25-7.

24 Snow N, Lucas AE, Grau M, Steiner M. Purulent mediastinal abscess secondary to Ludwig's angina. Arch Otolaryn-

25 Bounds GA. Subphrenic and mediastinal abscess formation: a complication of Ludwig's angina. Br $\mathcal{F}$ Oral Maxillofac Surg 1985; 23: 313-21.

26 Rubin MM, Cozzi GM. Fatal necrotizing mediastinitis as a complication of an odontogenic infection. $\mathcal{F}$ Oral Maxillofac Surg 1987; 45: 529-33.

27 Santos GH, Shapiro BM, Komisar A. Role of transoral irrigation in mediastinitis due to hypopharyngeal performation. Head Neck Surg 1986; 9: 116-21.

28 Levine TM, Wurster CF, Krespi YP. Mediastinitis occurring as a complication of odontogenic infections. Laryngoscope 1986; 96: 747-50.

29 Allen D, Loughnan TE, Ord RA. A reevaluation of the role of tracheostomy in Ludwig's angina. $\mathcal{F}$ Oral Maxillofac Surg of tracheostomy

30 Alexander DW, Leonard JR, Trail ML. Vascular complications of deep neck abscesses. Laryngoscope 1968; 78: $361-70$. 31 Roser SM, Chow AW, Brady FA. Necrotizing fasciitis. $\dot{f}$ Oral Surg 1977; 35: 730-2

32 Chow AW, Roser SM, Brady FA. Orofacial odontogenic infections. Ann Intern Med 1978; 88: 392-402.

33 Nakajima H, Seg H, Yokota T et al. Two cases of mediastinitis as complication of odontogenic infection and tonsilitis. Nippon Shikkan Gakkai Zaashi 1993; 31: 754-9.

34 Garaka CJ, Gay EG. Mediastinitis from odontogenic infection: report of three cases and review of the literature. Int $\mathcal{F}$ Oral Maxillofac Surg 1991; 20: 65-8.

35 Horowitz MD, Sosa JL, Lickstein DA. Descending necrotizing mediastinitis (Letter). Ann Thorac Surg 1990; 50: 859-60.

36 Spilka CJ. Pathways of dental infections. $f$ Oral Surg 1966; 24: 111-24. 